\title{
УСЛОВИЯ СХОДИМОСТИ ОДНОЙ ДВУХУРОВНЕВОЙ ИТЕРАЦИОННОИ СХЕМЫ ДЛЯ ВЫЧИСЛЕНИЯ УСТАНОВИВШИХСЯ РЕЖИМОВ ХИМИКО-ТЕХНОЛОГИЧЕСКИХ СИСТЕМ
}

A. ROOSE. OHE KEEMIAPROTSESSIDE TEHNOLOOGILISTE SUSTEEMIDE STATSIONAARSETE REZIIMIDE ARVUTAMISEKS ETTENAHTUD KAHENIVOOLISE ITERATSIOONIPROTSESSI KOONDUVUSEST

A. ROOSE. CONVERGENCE OF THE TWO-LEVEL ALGORITHM FOR CHEMICAL PLANT SIMULATION PROBLEMS

В [ $\left.{ }^{1}\right]$ показано, что модель статики любой химико-технологической системы (ХTC) может быть сконструирована в т. н. двухуровневом виде. В частности, она может иметь вид $\left[{ }^{2}\right]$

$$
\begin{aligned}
& x=A(z) y, \\
& F(x, z)=0,
\end{aligned}
$$

где (1а) - верхний уровень, выписанный исходя из условий материального баланса ХТС в целом; (1б) - совокупность моделей ключевых аппаратов в ХTC; $x$ - вектор всех входных потоков ключевых аппаратов в ХTC; $y$ - вектор потоков сырья в ХTC в целом; $z$ - вектор некоторых вспомогательных переменных; $A(z)$ - матричная функция, определенная на основе структуры рассматриваемой системы.

Проблема вычисления установившихся режимов ХТС в терминах модели (1) сводится к определению решения $\left(z^{*}, x^{*}\right)$ системы уравнений (1) при фиксированном векторе $y$. Для этого в $\left[{ }^{2}\right]$ применен следующий двухуровневой итерационный процесс:

$$
\begin{aligned}
& x_{n+1}=A\left(z_{n}\right) y, \\
& F\left(x_{n+1}, z_{n+1}\right)=0, \quad n=0,1, \ldots,
\end{aligned}
$$

где $z_{0}-$ начальное приближение.

Нетрудно видеть, что для каждого $n=0,1, \ldots$ система нелинейных уравнений (2б) должна быть решена заново. Отметим, что выражение (2) есть в сущности метод Гаусса-Зейделя для решения системы (1). Так как система нелинейных уравнений (2б) имеет специфическую структуру (см. подробнее в $\left.\left[{ }^{2}\right]\right)$, то для реализации на ЭВМ более удобен алгоритм (2), чем традиционные, т. н. последовательные алгоритмы вычисления установившихся режимов ХTC (см. $\left.\left[{ }^{3}\right]\right)$ или чем, например, алгоритм Ньютона-Канторовича для численного решения системы (1). 
Условия, гарантирующие сходимость процесса (2), определяет следующая

Т е орем а. Пусть определен элемент $z_{1},\|\cdot\|$ обозначает норму элемента (вектора или матрицы) $и$

1) $\|y\| \leqslant U$

2) матрица $A(z)$ однозначна и удовлетворяет условию Липшица $\|A(\mu)-A(\eta)\| \leqslant K\|\mu-\eta\| n p u$

$$
\mu, \eta \in S_{1}\left(z_{0}, \frac{1}{1-C}\left\|z_{1}-z_{0}\right\|\right) ;
$$

3) на прямом произведении сфер

$$
S_{1}\left(z_{0}, \frac{1}{1-C}\left\|z_{1}-z_{0}\right\|\right) \times S_{2}\left(x_{1}, K U \frac{1}{1-C}\left\|z_{1}-z_{0}\right\|\right)
$$

и в ее окрестности существуют непрерывные производные

$\frac{\partial F(x, z)}{\partial z}, \frac{\partial F(x, z)}{\partial x}$, обратный оператор $\left[\frac{\partial F(x, z)}{\partial z}\right]^{-1}=\Gamma$ и имеют место оценки $\|\Gamma\| \leqslant M,\left\|\frac{\partial F(x, z)}{\partial x}\right\| \leqslant N$;

4) $C=M N K U<1$.

Тогда итерационный процесс (2) определяет сходящиеся $\kappa$ решению $\left(z^{*}, x^{*}\right)$ системь (1) последовательности $\left\{z_{n}\right\}_{n=0}^{\infty} u\left\{x_{n}\right\}_{n=1}^{\infty}$ и имеют место оценки

$$
\begin{array}{ll}
\left\|z^{*}-z_{n}\right\| \leqslant \frac{C^{n}}{1-C}\left\|z_{1}-z_{0}\right\|, & n=0,1, \ldots, \\
\left\|x^{*}-x_{n}\right\| \leqslant K U \frac{C^{n-1}}{1-C}\left\|z_{1}-z_{0}\right\|, & n=1,2, \ldots,
\end{array}
$$

причем $\left(z^{*}, x^{*}\right)$ - единственное решение системь (1) в области

$$
S_{1}\left(z_{0}, \frac{1}{1-C}\left\|z_{1}-z_{0}\right\|\right) \times S_{2}\left(x_{1}, K U \frac{1}{1-C}\left\|z_{1}-z_{0}\right\|\right) .
$$

Доказательство теоремы полностью приводить не будем. План доказательства следующий. Если $F(x, z)=0$ определяет неявную функцию $z=\Phi(x)$, то процесс (2) можно переписать в виде

$$
z_{n+1}=\Phi\left[A\left(z_{n}\right) y\right]=\bar{\Phi}\left(z_{n}\right), \quad n=0,1, \ldots .
$$

Это обыкновенный итерационный процесс, для которого на основе принципа сжимающих отображений легко выписать условия сходимости последовательности $\left\{z_{n}\right\}_{n=0}^{\infty}$ к точному решению уравнения $z=$ $=\bar{\Phi}(z)\left[{ }^{4}\right]$. Единственная трудность состоит в том, что удовлетворяющая условию Липшица неявная функция $\Phi(x)$ должна быть определена в области, которая содержит всю последовательность $\left\{x_{n}\right\}_{n=1}^{\infty}$. Эта область вытекает из условий сходимости процесса (3). Условия существования неявной функции в наперед заданной области, в свою очередь, определяются нелокальной теоремой Лозинского $\left[{ }^{5}\right]$. Если эти условия добавить к условиям теоремы о сходимости процесса $z_{n+1}=$ $=\bar{\Phi}\left(z_{n}\right), n=0,1, \ldots$, то в результате получим теорему о сходимости процесса (2). Надо отметить, что это не единственно возможный под- 
ход к доказательству сходимости процесса (2). Приведенная выше методика имеет неконструктивный характер - она ничего не говорит о способах фактической реализации алгоритма (2), т. е. о способах решения уравнений (2б). Основываясь на методе вариации параметра $\left[{ }^{4,6}\right]$, можно дать и конструктивное доказательство приведенной выше теоремы. При этом проблема численного решения последовательности уравнений (2б) сводится к численному решению последовательности задач Коши

$$
\begin{gathered}
\frac{d z}{d t}=-\left\{\frac{\partial F\left[t x_{n+1}+(1-t) x_{n}, z\right]}{\partial z}\right\}^{-1} \frac{\partial F\left[t x_{n+1}+(1-t) x_{n}, z\right]}{\partial x}\left(x_{n+1}-x_{n}\right), \\
z(0)=z_{n}, \quad \mathrm{t} \in[0,1], \quad n=1,2, \ldots .
\end{gathered}
$$

При фиксированном $n=1,2, \ldots$ задача Коши (4) определяет в условиях теоремы приближение $z_{n+1}$, причем $z_{n}$ используется как начальное условие задачи Коши. Однако конструктивное доказательство теоремы из-за громоздкости обсуждать здесь не будем.

Алгоритм (1) был использован для вычисления установившихся режимов цеха производства карбамида при различных входных потоках сырья $\left[{ }^{2}\right]$.

\section{ЛИТЕР АТ У Р А}

1. Н аг и ев М. Ф., Основы химической кинетики промышленных систем, Баку, 1950.

2. T a vast, R., Roose, A., In: Proc. of the Symposium «Computers in the Design and Erection of Chemical Plants», Karlovy Vary, Czechoslovakia, Aug.-Sept., 1975, p. 443.

3. Eva ns, L. B., Stew a rd, D. G., S p r a g u e, C. R., Chem. Engng Progr., 64, No. 4, 39 (1968)

4. Ortega, J. M., Rhein boldt, W. C., Iterative Solution of Nonlinear Equations in Several Variables, New York, 1970.

5. Лози н с ки й С. М., Вестник ЛГУ, № 7,131 (1957).

6. Д а в и ден ко Д. Ф., Докл. АН СССР, 88, № 4, 601 (1953).

Ннститут кибернетики

Академии наук Эстонской ССР
Поступила в редакцию 27/IX 1977 